\title{
Origin of the piezochromism in $\mathrm{Cs}_{2} \mathrm{CuCl}_{4}$ : Electron-phonon and crystal-structure correlations
}

\author{
E. Jara, J. A. Barreda-Argüeso, J. González, and F. Rodríguez* \\ MALTA TEAM, DCITIMAC, Facultad de Ciencias, Universidad de Cantabria, 39005 Santander, Spain \\ R. Valiente \\ Nanomedicine Group-IDIVAL, Dpto. Física Aplicada, Facultad de Ciencias, Universidad de Cantabria, 39005 Santander, Spain
}

(Received 30 January 2019; revised manuscript received 29 March 2019; published 18 April 2019)

\begin{abstract}
By using optical absorption and Raman spectroscopy, we have investigated the effects of pressure in the structural, electronic, and vibrational properties of $\mathrm{Cs}_{2} \mathrm{CuCl}_{4}$ in the $0-20 \mathrm{GPa}$ range. Our study has provided a complete characterization of the electronic and vibrational structures of $\mathrm{Cs}_{2} \mathrm{CuCl}_{4}$ in the low-pressure Pnma phase as a function of cell volume and the $\mathrm{Cu}-\mathrm{Cl}$ bond length, $R_{\mathrm{Cu}-\mathrm{Cl}}$. We have focused on the electronic structure in the Pnma phase, which is mainly related to the tetrahedral $\mathrm{CuCl}_{4}^{2-}$ units distorted by the Jahn-Teller effect. The strong piezochromism of $\mathrm{Cs}_{2} \mathrm{CuCl}_{4}$ is caused by the opposite shift of the charge-transfer band gap and the $\mathrm{Cu}^{2+} d$ - $d$ crystal-field transitions with pressure. The high-pressure structure of $\mathrm{Cs}_{2} \mathrm{CuCl}_{4}$ above $4.9 \mathrm{GPa}$ yields structural transformations that we ascribe to a change of coordination around $\mathrm{Cu}^{2+}$. The high-pressure phase appears largely amorphized, therefore any structural information from x-ray diffraction is ruled out. Here we use electronic and vibrational probes to get inside the structural information.
\end{abstract}

DOI: 10.1103/PhysRevB.99.134106

\section{INTRODUCTION}

$\mathrm{Cs}_{2} \mathrm{CuCl}_{4}$ (orthorhombic Pnma at ambient conditions) is a wide-band-gap charge-transfer (CT) semiconductor. Under pressure it exhibits a puzzling optical behavior which is associated with the $\mathrm{Cu}^{2+}$ absorption and its structural changes [1]. Both $\mathrm{Cl}^{-} \rightarrow \mathrm{Cu}^{2+} \mathrm{CT}$ and $d-d$ absorption bands undergo unusually large pressure shifts and intensity changes showing abrupt jumps at about $5 \mathrm{GPa}$. These shifts are responsible for the color change exhibited by the crystal as a function of pressure (piezochromism). The characteristic yellow-orange color of this crystal at ambient conditions is mainly defined by the tail of the CT band (band gap) placed around $450 \mathrm{~nm}$ [2]. The low-lying CT band gap, and thus its yellow-orange color are a consequence of the isolated $\mathrm{CuCl}_{4}^{2-}$ tetrahedra units in the Pnma phase, which shows a flattened $\left(D_{2 d}\right)$ distortion by the Jahn-Teller (JT) effect. Unlike $\mathrm{Cs}_{2} \mathrm{CoCl}_{4}$, the JT distortion splitting of the $d$ - $d$ bands of $\mathrm{Cu}^{2+}\left(3 d^{9}\right)$ does not affect the color as they appear at 1110 and $1820 \mathrm{~nm}$ in the nearinfrared range [2,3]. The isolated character of $\mathrm{CuCl}_{4}^{2-}$ in its Pnma phase has been clearly revealed by Raman spectroscopy through the characteristic internal modes at $\Gamma$ point of a distorted tetrahedron $\left(D_{2 d}\right)$. It constitutes an efficient probe for local and crystal structures [4-6]. The study of electronic, vibrational, and crystal structures under high-pressure conditions of this relatively low compressible materials ( $\beta=$ $0.067 \mathrm{GPa}^{-1}-$, i.e., $K_{0}=15 \mathrm{GPa}-$ ) [7] allows us to establish structural correlations to understand (i) the electronic properties of $\mathrm{Cu}^{2+}$ in tetrahedral coordination in less compressible oxides and (ii) how a lattice of independent $\mathrm{CuCl}_{4}^{2-}$ units under compression evolves toward denser phases.

\footnotetext{
*fernando.rodriguez@unican.es
}

The variation of the crystal structure of $\mathrm{Cs}_{2} \mathrm{CuCl}_{4}$ and $\mathrm{Cs}_{2} \mathrm{CoCl}_{4}$ under pressure has been previously investigated by $\mathrm{X}$-ray diffraction (XRD) in the $0-5 \mathrm{GPa}$ range, where both crystals are in the Pnma crystallograpic phase [7]. A structural phase transition in $\mathrm{Cs}_{2} \mathrm{CuCl}_{4}$ takes place just above $5 \mathrm{GPa}$, yielding a deep color change from yelloworange to black. This high-pressure phase (HP) could not be identified by XRD due to amorphization [7]. The crystal structure (polymorphism) strongly determines the optical properties of $\mathrm{Cu}^{2+}$ chlorides like $\mathrm{Cs}_{2} \mathrm{CuCl}_{4}$. Particularly, the $\mathrm{Cu}^{2+}$ coordination-symmetry and crystal-field strengthand the way $\mathrm{Cu}^{2+}$ ions are coupled to each other, i.e., either as isolated units or as interconnected $\mathrm{Cu}-\mathrm{Cu}$ polyhedra sharing $\mathrm{Cl}^{-}$ligand [8,9]. Therefore, the knowledge of how these links and crystal-field effects influence the optical and Raman spectra is important to extract structural information from the electronic and/or vibrational spectra at high-pressure conditions. Thus, the establishment of correlations between structure and electronic and vibrational properties becomes essential to achieve this goal [3]. In this paper, the relationship between dihedral $\mathrm{Cl}-\mathrm{Cu}-\mathrm{Cl}$ angle of the JT-distorted flattened tetrahedra and the $\mathrm{Cu}^{2+} d$-orbital splitting experimentally obtained by optical absorption spectroscopy and its pressure dependence is investigated. These correlations will be used to analyze the shifts experienced by the band-gap energy and $d$ - $d$ bands as well as the Raman peaks with pressure in the $\mathrm{Cs}_{2} \mathrm{CuCl}_{4}$ Pnma phase, and how they change above the structural phase transition observed $5 \mathrm{GPa}$.

\section{EXPERIMENTAL}

Single crystals of $\mathrm{Cs}_{2} \mathrm{CuCl}_{4}$ were grown by slow evaporation at $30^{\circ} \mathrm{C}$ from acidic $(\mathrm{HCl})$ solution containing a 2:1 stoichiometric ratio of the $\mathrm{CsCl}$ and $\mathrm{CuCl}_{2} \cdot \mathrm{H}_{2} \mathrm{O}$. The ambient 
pressure crystal structure, Pnma space group, was checked by XRD on powder samples using a Bruker D8 Advance diffractometer. The measured cell parameters at ambient conditions were $a=9.770 \AA, b=7.617 \AA, c=12.413 \AA$.

A Boehler-Almax diamond anvil cell (DAC) was used for the high-pressure studies. 200- $\mu \mathrm{m}$-thickness Inconel 625 gaskets were preindented and suitable $200-\mu \mathrm{m}$-diameter holes were perforated with a BETSA motorized electrical discharge machine. Given that $\mathrm{Cs}_{2} \mathrm{CuCl}_{4}$ is soluble in common pressure transmitting medialike methanol-ethanol-water (16:4:1), spectroscopic paraffin oil (Merck) was used as alternative pressure transmitting media. It must be noted, however, that according to the ruby line broadening, nonhydrostatic effects were significant in the explored range, as previously reported [10].

The microcrystals used in pressure experiments for Raman spectroscopy and optical absorption were extracted from $\mathrm{Cs}_{2} \mathrm{CuCl}_{4}$ single crystal. Due to the strong sample absorption outside the transparency window (500-700 nm) we selected laser wavelengths within this window and kept the power of the laser beam below $2 \mathrm{~mW}$ to avoid burning of the sample as it can be easily recognized by the black spot on the crystal surface. The Raman spectra were taken with a Horiba T64000 triple spectrometer using the 514.5-nm and 647-nm lines of a Coherent Innova Spectrum $70 \mathrm{Ar}^{+}-\mathrm{Kr}^{+}$ laser and nitrogen-cooled CCD (Jobin-Yvon Symphony) with a confocal microscope for detection. For measuring the low frequency modes, we used the triple monochromator in the subtractive configuration.

The experimental setup for room temperature optical absorption measurements with the DAC has been described elsewhere [10-13]. VIS-NIR absorption data were obtained by using an Ocean Optics USB2000+ spectrometer and a NIRQUEST 512 spectrometer, respectively. For the FIR measurements, a Thermo Nicolet Contin $\mu$ um was used. Pressure was calibrated using the fluorescence ruby method. Suitable single crystal plates of $\mathrm{Cs}_{2} \mathrm{CuCl}_{4}(150 \mu \mathrm{m}$ thickness) were used for obtaining the absorption coefficient in the CT-band gap tail and onset of the NIR absorption using a Cary 6000i spectrophotometer. The thickness of the microcrystal inside the DAC was determined through the absorption spectra using the absorption coefficient determined macroscopically.

\section{RESULTS AND DISCUSSION}

\section{A. Raman spectroscopy of $\mathrm{Cs}_{2} \mathrm{CuCl}_{4}$}

\section{Raman spectrum at ambient conditions}

Figure 1 compares the Raman spectrum of $\mathrm{Cs}_{2} \mathrm{CuCl}_{4}$ at ambient pressure with the isomorphous $\mathrm{Cs}_{2} \mathrm{CoCl}_{4}$ [3]. The

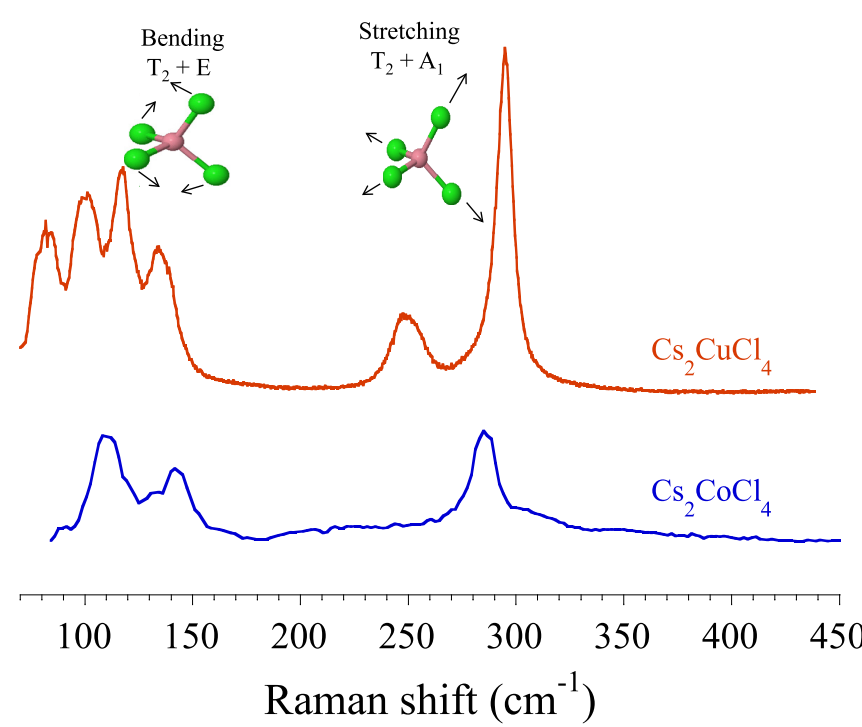

FIG. 1. Raman spectra of $\mathrm{Cs}_{2} \mathrm{CuCl}_{4}$ and $\mathrm{Cs}_{2} \mathrm{CoCl}_{4}$ at ambient conditions showing the high frequency optical modes corresponding to internal vibrations of the $M \mathrm{Cl}_{4}^{2-}(M=\mathrm{Cu}, \mathrm{Co})$ tetrahedra in the orthorhombic Pnma phase.

corresponding peak frequencies and mode assignment are collected in Table I. The Raman peak assignment has been done on the basis of previous measurements reported elsewhere [3,14-16]. The observed peaks in $\mathrm{Cs}_{2} \mathrm{CuCl}_{4}$ mainly correspond to internal modes of the $\mathrm{CuCl}_{4}^{2-}$ tetrahedra according to assignments given in Table I $[3,5,15]$. The first set corresponds to $\mathrm{Cs}_{2} \mathrm{CuCl}_{4}$ lattice modes $\left(81 \mathrm{~cm}^{-1}\right)$ [15] and to internal $\mathrm{Cl}-\mathrm{Cu}-\mathrm{Cl}$ bending modes of $\mathrm{E}$ and $\mathrm{T}_{2}$ symmetry in $T_{d}\left(\mathrm{~B}_{1}+\mathrm{A}_{1}\right.$ and $\mathrm{E}+\mathrm{B}_{2}$ in $\left.D_{2 d}\right): v_{2}\left(\mathrm{~B}_{1}+\mathrm{A}_{1} / \mathrm{E}\right)=104$ $\mathrm{cm}^{-1}, v_{4^{\prime}}\left(\mathrm{B}_{2} / \mathrm{T}_{2}\right)=120 \mathrm{~cm}^{-1}$, and $v_{4^{\prime \prime}}\left(\mathrm{E} / \mathrm{T}_{2}\right)=137 \mathrm{~cm}^{-1}$, respectively. The main peaks of the second set correspond to internal $\mathrm{Cu}-\mathrm{Cl}$ stretching modes of $\mathrm{A}_{1}$ and $\mathrm{T}_{2}$ in $T_{d}\left(\mathrm{~A}_{1}\right.$, and $\mathrm{E}+\mathrm{B}_{2}$, respectively, in $\left.D_{2 d}\right): v_{1}\left(\mathrm{~A}_{1} / \mathrm{A}_{1}\right)=295 \mathrm{~cm}^{-1}$, $v_{3^{\prime \prime}}\left(\mathrm{B}_{2} / \mathrm{T}_{2}\right)=279 \mathrm{~cm}^{-1}$, and $\nu_{3^{\prime}}\left(\mathrm{E} / \mathrm{T}_{2}\right)=249 \mathrm{~cm}^{-1}$. These peaks compare well with those measured for $\mathrm{CoCl}_{4}^{2-}\left(T_{d}\right)$ in the isostructural $\mathrm{Cs}_{2} \mathrm{CoCl}_{4}$ compound [3]; however, there is a significant difference between the Raman spectra of $\mathrm{Cs}_{2} \mathrm{CoCl}_{4}$ and $\mathrm{Cs}_{2} \mathrm{CuCl}_{4}$ - that is, the splitting of some peaks in the latter compound that we associate with the lower local symmetry of $\mathrm{CuCl}_{4}^{2-}\left(D_{2 d}\right)$ due to the JT effect. This effect is clearly seen in the parent $T_{d}$ peaks $v_{3}\left(\mathrm{~T}_{2}\right)$ and $v_{4}\left(\mathrm{~T}_{2}\right)$, which split into $v_{3^{\prime}}\left(\mathrm{E} / \mathrm{T}_{2}\right)+v_{3^{\prime \prime}}\left(\mathrm{B}_{2} / \mathrm{T}_{2}\right)$, and to a lesser extent in $v_{4^{\prime}}\left(\mathrm{B}_{2} / \mathrm{T}_{2}\right)$ $+v_{4 \prime}\left(\mathrm{E} / \mathrm{T}_{2}\right)$, respectively (Table I). A salient differential feature between both isomorphous compounds is the solely presence of the stretching split mode $\nu_{3^{\prime}}\left(\mathrm{E} / \mathrm{T}_{2}\right)$ at $249 \mathrm{~cm}^{-1}$

TABLE I. Comparison of the bending and stretching modes for $(M=\mathrm{Cu}, \mathrm{Co})$. Peak assignment of $\mathrm{Cs}_{2} \mathrm{CuCl}_{4} \mathrm{modes}$ in $T_{d}$ and $D_{2 d}$ symmetries and corresponding vibrational frequencies are given in Table II [3,14-16].

\begin{tabular}{|c|c|c|c|c|c|c|}
\hline $\mathrm{Cs}_{2} M \mathrm{Cl}_{4}$ & $v_{2}\left(\mathrm{~B}_{1}+\mathrm{A}_{1} / \mathrm{E}\right) / \mathrm{cm}^{-1}$ & $v_{4^{\prime}}\left(\mathrm{B}_{2} / \mathrm{T}_{2}\right) / \mathrm{cm}^{-1}$ & $v_{4^{\prime \prime}}\left(\mathrm{E} / \mathrm{T}_{2}\right) / \mathrm{cm}^{-1}$ & $v_{3^{\prime}}\left(\mathrm{E} / \mathrm{T}_{2}\right) / \mathrm{cm}^{-1}$ & $v_{3^{\prime \prime}}\left(\mathrm{B}_{2} / \mathrm{T}_{2}\right) / \mathrm{cm}^{-1}$ & $v_{1}\left(\mathrm{~A}_{1} / \mathrm{A}_{1}\right) / \mathrm{cm}^{-1}$ \\
\hline $\mathrm{Co}[3]$ & 112 & - & 142 & 315 & - & 287 \\
\hline
\end{tabular}



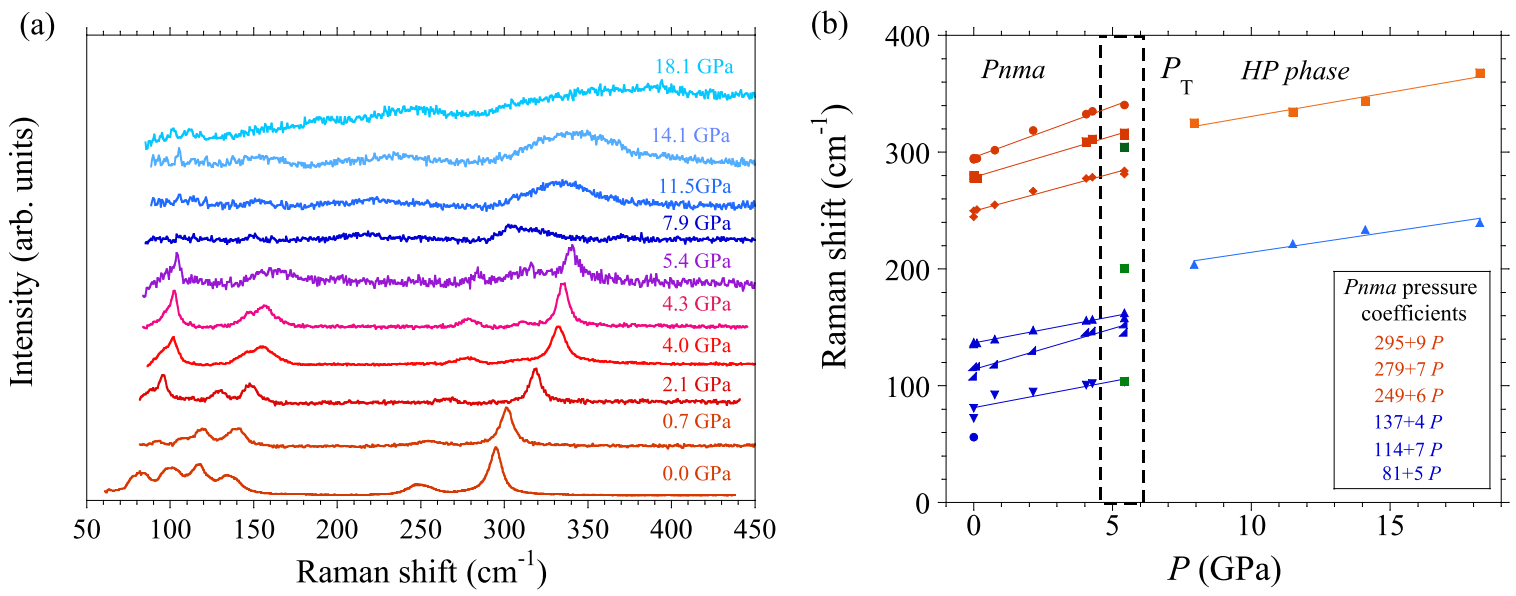

FIG. 2. (a) Pressure dependence of the Raman spectrum of $\mathrm{Cs}_{2} \mathrm{CuCl}_{4}$. Note the pressure-induced phase transition at $5.4 \mathrm{GPa}$. (b) Variation of the vibrational frequencies with pressure. The green squares correspond to measurements at the phase-coexistence pressure at $5.4 \mathrm{GPa}$. The least-squares linear fits to the experimental frequencies in the Pnma are given in the inset. The frequencies, corresponding pressure shifts and Grüneinsen parameters are collected in Table II.

in the Raman spectrum of $\mathrm{Cs}_{2} \mathrm{CuCl}_{4}$. This mode constitutes the fingerprint of the $D_{2 d}$ JT distortion in $\mathrm{CuCl}_{4}^{2-}$.

\section{Pressure dependence}

The variation of the Raman spectrum of $\mathrm{Cs}_{2} \mathrm{CuCl}_{4}$ with pressure is shown in Fig. 2. All Raman frequencies increase linearly with pressure at a rate ranging from 4.5 to $8.7 \mathrm{~cm}^{-1} \mathrm{GPa}^{-1}$ in its Pnma phase in the $0-5 \mathrm{GPa}$ range (Table II). There is no significant difference between the absolute values of the pressure shifts in the bending and stretching modes-what makes the relative variation for the bending modes larger than for the stretching modes, as it is clearly reflected by the obtained Grüneisen parameter of each mode: $\gamma=0.49$ and 1.0 for the $\nu_{4^{\prime \prime}}\left(\mathrm{E} / \mathrm{T}_{2}\right)$ and $\nu_{2}\left(\mathrm{~B}_{1}+\right.$ $\left.\mathrm{A}_{1} / \mathrm{E}\right)$ bending modes, respectively, and $\gamma\left(A_{1} / A_{1}\right)=0.44$, $\gamma\left(\mathrm{B}_{2} / \mathrm{T}_{2}\right)=0.38$, and $\gamma\left(\mathrm{E} / \mathrm{T}_{2}\right)=0.39$ for the stretching modes. Although $\gamma$ values seem to be lower than the expected ones for stretching modes $(\gamma \approx 2-3)$ [17], it is a direct consequence of the larger bulk compressibility of $\mathrm{Cs}_{2} \mathrm{CuCl}_{4}$ with respect to the local compressibility of $\mathrm{CuCl}_{4}^{2-}$. Given that these Grüneisen parameters are obtained by using the crystal bulk modulus $\left(K_{0}=15 \mathrm{GPa}\right)$ [7], they change when we refer these pressure shifts to the local bulk modulus of $\mathrm{CuCl}_{4}^{2-}$ instead. Thus, the local Grüneisen parameters of the stretching modes are then $\gamma_{\mathrm{loc}}\left(A_{1}\right) \cong 3.4$, and $\gamma_{\mathrm{loc}}\left(\mathrm{B}_{2} / \mathrm{T}_{2}\right) \cong 2.9$, and $\gamma_{\text {loc }}\left(\mathrm{E} / \mathrm{T}_{2}\right) \cong 3.0$, using a local bulk modulus for $\mathrm{CuCl}_{4}^{2-}$ of $K_{\text {loc }}=115 \mathrm{GPa}$, which is derived from the $\mathrm{Cs}_{2} \mathrm{CuCl}_{4}$ structural data [7]. These parameters are similar to those expected for high-frequency stretching modes. It is worth noting that the $\mathrm{Cs}_{2} \mathrm{CuCl}_{4}$ Grüneisen parameters are slightly higher than those observed in $\mathrm{Cs}_{2} \mathrm{CoCl}_{4}$ in spite of the pressure coefficients of the Raman peaks being similar in both compounds. This is due to the smaller bulk modulus of $\mathrm{Cs}_{2} \mathrm{CuCl}_{4}\left(K_{0}=\right.$ $15 \mathrm{GPa})$ in comparison to $\mathrm{Cs}_{2} \mathrm{CoCl}_{4}\left(K_{0}=21 \mathrm{GPa}\right)$ [3]. The $D_{2 d}$ bending distortion of $\mathrm{CuCl}_{4}^{2-}$ probably makes $\mathrm{Cs}_{2} \mathrm{CuCl}_{4}$ compress easier than the slightly denser cobalt compound. On the other hand, the $\mathrm{CuCl}_{4}^{2-}$ local bulk modulus is higher than $\mathrm{CoCl}_{4}^{2-}$ probably due to the compression process attained in the distorted tetrahedra yielding bend-angle reduction accompanied by a smaller bond distance compression, both effects contributing to a smaller local volume reduction with pressure. The frequency, pressure coefficient, and Grüneisen parameter of the observed Raman modes are collected in Table II.

The Raman spectrum completely changes above $5.4 \mathrm{GPa}$, thus unveiling a structural phase transition. Above this pressure, the spectrum is poorly resolved, being compatible with a strongly amorphized phase as already pointed out from XRD data [7]. Nevertheless, we still observe two broad peaks at 200 and $320 \mathrm{~cm}^{-1}$, indicating that the HP likely involves a change

TABLE II. Experimental Raman frequency of the vibrational optical modes of $\mathrm{Cs}_{2} \mathrm{CuCl}_{4}$ at $T=290 \mathrm{~K}$ obtained from Figs. 1 and 2 . The lattice modes correspond to internal modes of $\mathrm{CuCl}_{4}^{2-}$ and external lattices modes, and are labeled according to $D_{2 d} / T_{d}$ symmetry irreps following the assignment given elsewhere [15]. Their pressure shift rate as well as the corresponding Grüneisen parameter associated with the crystal volume $(\gamma)$ and the tetrahedron volume $\left(\gamma_{\text {loc }}\right)$ have been derived from the corresponding crystal and local bulk moduli: $K_{0}=15 \mathrm{GPa}$ and $K_{\mathrm{loc}}=115 \mathrm{GPa}$.

\begin{tabular}{|c|c|c|c|c|c|c|c|}
\hline \multirow[t]{2}{*}{ Raman modes $\mathrm{CuCl}_{4}^{2-}$} & & \multicolumn{3}{|c|}{ Bending $\mathrm{Cu}-\mathrm{Cl}-\mathrm{Cu}$ mode } & \multicolumn{3}{|c|}{ Stretching $\mathrm{Cu}-\mathrm{Cl}$ mode } \\
\hline & Lattice modes & $v_{2}\left(B_{1}+A_{1} / E\right)$ & $v_{4^{\prime}}\left(B_{2} / T_{2}\right)$ & $v_{4^{\prime \prime}}\left(E / T_{2}\right)$ & $\nu_{3^{\prime}}\left(E / T_{2}\right)$ & $v_{3^{\prime \prime}}\left(B_{2} / T_{2}\right)$ & $v_{1}\left(A_{1}\right)$ \\
\hline$v\left(\mathrm{~cm}^{-1}\right)$ & 81 & 104 & 120 & 137 & 249 & 279 & 295 \\
\hline$d v / d P\left(\mathrm{~cm}^{-1} \mathrm{GPa}^{-1}\right)$ & 4.5 & 7.0 & 7.0 & 4.5 & 6.5 & 7.1 & 8.7 \\
\hline$\gamma=\left(K_{0} / v\right) d v / d P$ & 0.83 & 1.0 & 0.88 & 0.49 & 0.39 & 0.38 & 0.44 \\
\hline$\gamma_{\mathrm{loc}}=\left(K_{\mathrm{loc}} / v\right) d v / d P$ & - & 7.7 & 6.7 & 3.8 & 3.0 & 2.9 & 3.4 \\
\hline
\end{tabular}




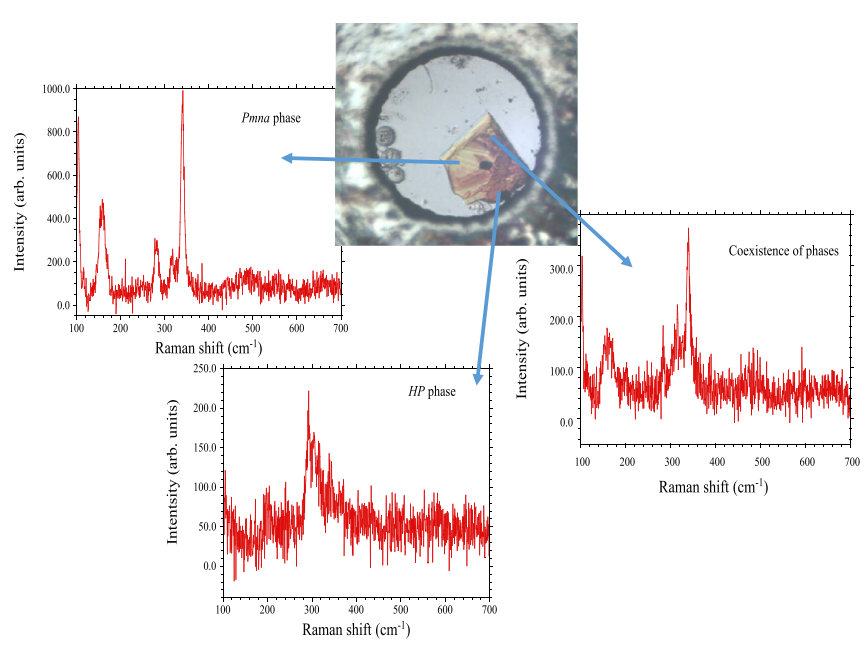

FIG. 3. Image of a single crystal of $\mathrm{Cs}_{2} \mathrm{CuCl}_{4}$ in a DAC at $5.4 \mathrm{GPa}$ showing different phase zones. The Raman spectrum of each crystal zone is indicated. Note how the crystal color varies from yellow to deep orange along different zones and its relationship with the Raman spectrum. Single crystal dimensions: $67 \times 67 \times 21 \mu \mathrm{m}^{3}$.

of $\mathrm{Cu}^{2+}$ coordination similar to $\mathrm{Cs}_{2} \mathrm{CoCl}_{4}$ [3]. Although the $\mathrm{HP}$ has not yet been identified, the presence of ligand-sharing $\mathrm{CuCl}_{6}^{4-}$ octahedra is likely. Interestingly, the $P n m a \rightarrow \mathrm{HP}$ phase transition is of first-order character with an associated hysteresis of $2 \mathrm{GPa}$. This character is also confirmed by the phase coexistence observed at $5.4 \mathrm{GPa}$ (Fig. 3), thus stressing the deep structural changes (volume and coordination polyhedra) attained in the HP phase.

\section{Temperature dependence of Raman spectra}

The temperature dependence of the optical $v_{1}\left(A_{1}\right)$ phonon has been studied in the $20-600 \mathrm{~K}$ range at ambient pressure. Figure 4(a) shows the variation of the Raman spectrum with temperature and Fig. 4(b) the fit of the main $v_{1}\left(A_{1}\right)$ peak to a hyperbolic cotangent function, which is known to describe phenomenologically the temperature-induced spectral shifts

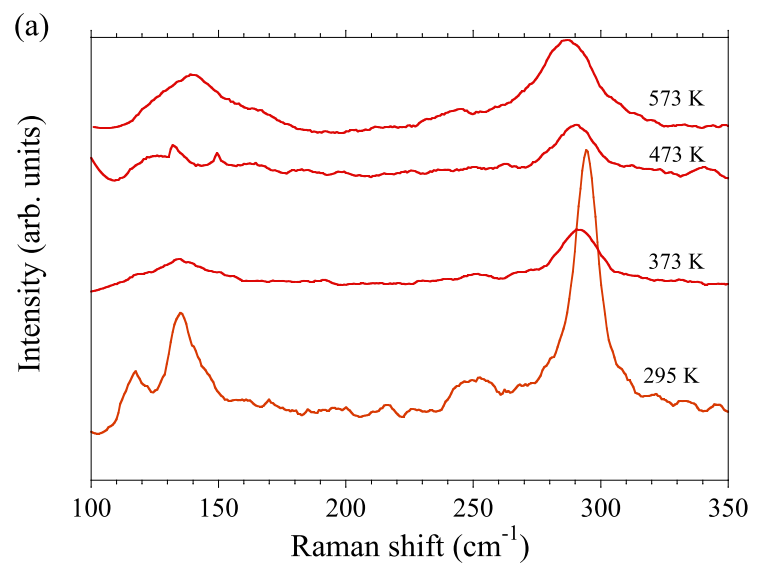

on the basis of an effective phonon frequency model [18]. The experimental thermal shift at room temperature (above $290 \mathrm{~K}$ ) is $(\partial v / \partial T)_{P}=-0.032 \mathrm{~cm}^{-1} \mathrm{~K}^{-1}$. It came to our attention that the peaks $v_{4^{\prime \prime}}\left(B_{2} / T_{2}\right)$ and $v_{1}\left(A_{1}\right)$ largely broaden while $\nu_{3^{\prime}}\left(E / T_{2}\right)$ disappears with temperature, making it difficult to obtain the thermal shift of $v_{4^{\prime \prime}}\left(B_{2} / T_{2}\right)$ and $v_{3^{\prime}}\left(E / T_{2}\right)$ and thus we only follow $v_{1}\left(A_{1}\right)$. To understand the important shift undergone by the $v_{1}\left(A_{1}\right)$ mode, we must analyze the different contributions-intrinsic and extrinsic ones-to the thermal shift for which the knowledge of the pressure-induced shift for this mode becomes essential.

The decrease of the Raman shift of the optical phonons with increasing temperature is due to two main contributions: change of frequency due to volume expansion (intrinsic contribution) and anharmonic coupling to phonons of other branches (extrinsic contribution). The real part of the phonon self-energy shift can be written as $[19,20]$

$$
v(T)=v_{0}+\Delta v_{1}(T)+\Delta v_{2}(T),
$$

where $\Delta v_{1}(T)$ is the thermal expansion contribution to the line shift and $\Delta v_{2}(T)$ is due to anharmonic coupling. $\Delta v_{1}(T)$ can be written as [19]

$$
\Delta v_{1}(T)=v_{0}\left\{\exp \left[-\gamma \int_{0}^{T} \alpha(T) d T\right]-1\right\},
$$

with $\gamma$ being the Grüneisen parameter for the optical Raman mode and $\alpha(T)$ the volume thermal expansion coefficient [21].

The correction of the self-energy due to anharmonic coupling is just a pure temperature effect for constant volume, and can be modeled as [19]

$$
\begin{aligned}
\Delta v_{2}(T)= & C\left[1+\frac{2}{\left(e^{x}-1\right)}\right] \\
& +D\left[1+\frac{3}{\left(e^{y}-1\right)}+\frac{3}{\left(e^{y}-1\right)^{2}}\right],
\end{aligned}
$$

where the first and second terms correspond to the coupling of the optical phonon to two identical phonons (cubic anharmonicities) and to three identical phonons (quadratic

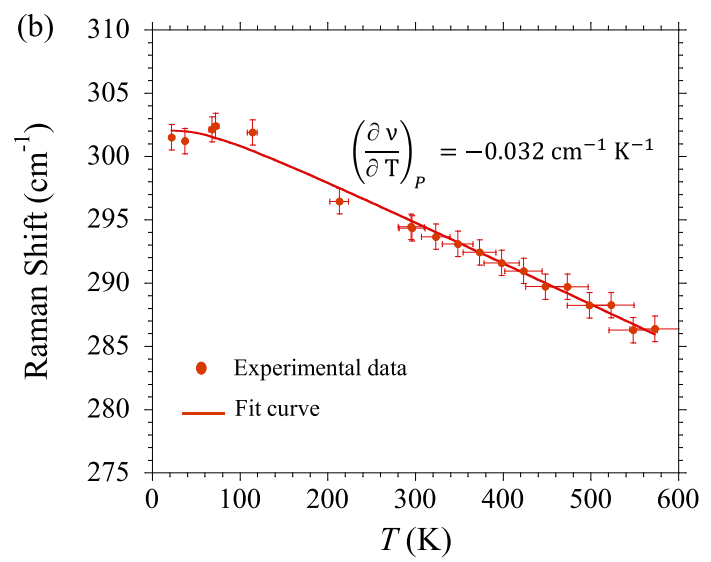

FIG. 4. (a) Temperature dependence of the Raman spectrum of $\mathrm{Cs}_{2} \mathrm{CuCl}_{4}$. (b) Experimental $v_{1}\left(A_{1}\right)$ peak frequencies as a function of temperature. Solid line corresponds to the fit of Raman shifts $v(T)$ to a phenomenological hyperbolic cotangent function: $v(T)=v(0)-$ $\Delta /\left(\mathrm{e}^{h v_{\text {eff }} / K_{B} T}-1\right)$ with $v(0)=302.0 \mathrm{~cm}^{-1}, \Delta=5.6 \mathrm{~cm}^{-1}$, and an effective vibrational frequency $v_{\text {eff }}=122.6 \mathrm{~cm}^{-1}$. The thermal coefficient of the $v_{1}\left(A_{1}\right)$ frequency at room temperature is $(\partial v / \partial T)_{P}=-0.032 \mathrm{~cm}^{-1} \mathrm{~K}^{-1}$. 


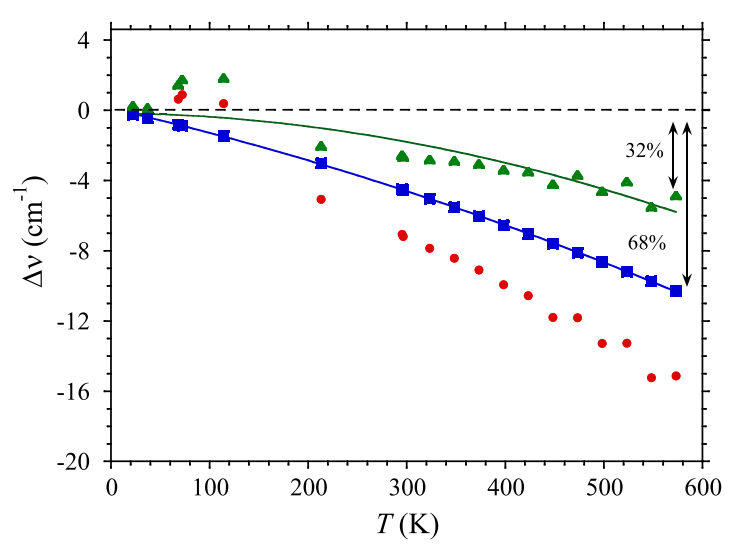

FIG. 5. Experimental thermal shift, $\Delta v(T)=v(T)-v(0)$ with $v(0)=302.0 \mathrm{~cm}^{-1}$ for the $v_{1}\left(A_{1}\right)$ mode at ambient pressure (red solid circles); thermal expansion contribution (blue solid squares); anharmonic phonon coupling contribution (green solid triangles). The thermal expansion contribution has been obtained through Eq. (2) while the phonon coupling contribution corresponds to the difference between the experimental line shift of Fig. 4 and the thermal expansion contribution (blue line). The green line is the fit of the phonon-coupling points to Eq. (1).

anharmonicities), respectively. Using the experimental variation of frequency with temperature given in Fig. 4(b) and the calculated thermal-expansion contribution to the shift through Eq. (2), we obtain the anharmonic-phonon-coulping term $\left[\Delta v_{2}(T)\right]$ as the difference between experimental results and the thermal-expansion contribution $\left[\Delta v_{1}(T)\right]$. The two contributions to the thermal shift are shown in Fig. 5. The phononcoupling contribution was then fitted to Eq. (3), taking $C$ and $D$ as fitting parameters since $x=h v_{0} / 2 K_{B} T$ and $y=$ $h v_{0} / 3 K_{B} T$ are calculated using $h v_{0}=302 \mathrm{~cm}^{-1}\left(h v_{0} / K_{B}=\right.$ $435 \mathrm{~K})$. At ambient pressure, we obtain $C=-0.11 \mathrm{~cm}^{-1}$ and $D=-0.10 \mathrm{~cm}^{-1}$. Both parameters are negative and have similar values. It means that both cubic and quadratic anharmonic phonon-coupling interactions equally contribute to the extrinsic thermal frequency shift of the totally symmetric Raman mode.

Figure 5 shows that the main contribution to the Raman shift is due to the thermal expansion which is of $68 \%$, whereas the phonon-coupling contribution represents only $32 \%$ at high temperature. Nevertheless, the phonon-coupling contribution slightly increases up to $\sim 36 \%$ at low temperature at the expense of the thermal expansion contribution being of $\sim 64 \%$. We conclude that the more important contribution to the frequency shift at ambient pressure is the thermal expansion contribution being twice the phonon-coupling contribution.

These relative contributions can be easily determined at room temperature from the experimental value of the derivative of the frequency shift with respect to temperature, $(\partial \nu / \partial T)_{P}=-0.032 \mathrm{~cm}^{-1} \mathrm{~K}^{-1}$. The intrinsic contribution can be calculated from the thermal expansion coefficient, $\alpha=$ $1.54 \times 10^{-4} \mathrm{~K}^{-1}$, and the bulk modulus, $K_{0}=15 \mathrm{GPa}$, by $(\partial v / \partial T)_{\text {intr }}=(\partial v / \partial P)_{290 K} \alpha K_{0}=-0.020 \mathrm{~cm}^{-1} \mathrm{~K}^{-1}$, which means a $63 \pm 4 \%$ of the total contribution in fair agreement with calculations performed for $v(T)$ in the whole temperature range.

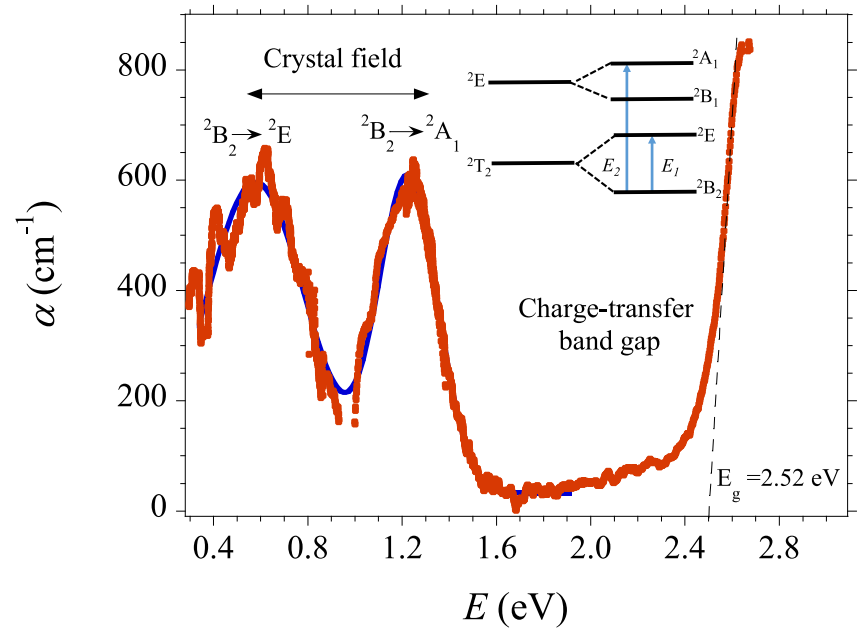

FIG. 6. Optical absorption spectrum of $\mathrm{Cs}_{2} \mathrm{CuCl}_{4}$ at ambient conditions. The blue line represents the fitting of the experimental points (in red) to the sum of two Gaussians. The crystal-field bands correspond to the two electric-dipole $D_{2 d}$-allowed $d$ - $d$ intraconfigurational transitions: ${ }^{2} \mathrm{~B}_{2} \rightarrow{ }^{2} \mathrm{E}(0.55 \mathrm{eV})$ and ${ }^{2} \mathrm{~A}_{1}(1.3 \mathrm{eV})$. The $\mathrm{Cl}^{-} \rightarrow \mathrm{Cu}^{2+} \mathrm{CT}$ band gap is $E_{g}=2.52 \mathrm{eV}$.

\section{B. Electronic structure, optical absorption spectra, and piezochromism of $\mathrm{Cs}_{2} \mathrm{CuCl}_{4}$}

At ambient conditions, the optical absorption spectrum of $\mathrm{Cs}_{2} \mathrm{CuCl}_{4}$ in the Pnma phase shows two intense bands in the near infrared, which are associated with $d$ - $d$ electronic transitions within the $\mathrm{CuCl}_{4}^{2-}\left(D_{2 d}\right)$ and an absorption threshold associated with the ligand-to-metal CT band in the visible, which is responsible for the band gap and the concomitant yellow-orange color of this crystal (Fig. 6). Peaks are assigned according to tetrahedral crystal-field transitions of $\mathrm{CuCl}_{4}^{2-}$, using both $T_{d}$ and $D_{2 d}$ irreps notation [22]. The two main peaks correspond to spin-allowed $d$ - $d$ electric-dipole transitions from the ${ }^{2} \mathrm{~B}_{2}$ ground state to the ${ }^{2} \mathrm{E}$, denoted as $E_{1}$ and ${ }^{2} \mathrm{~A}_{1}\left(E_{2}\right)$ excited states, and are located at 0.55 and $1.3 \mathrm{eV}$, respectively. The first transition ${ }^{2} \mathrm{~B}_{2} \rightarrow{ }^{2} \mathrm{E}$ is associated with the tetrahedral ${ }^{2} \mathrm{~T}_{2}$ splitting into ${ }^{2} \mathrm{~B}_{2}+{ }^{2} \mathrm{E}$ due to the $D_{2 d}$ JT distortion with ${ }^{2} \mathrm{~B}_{2}$ being the electronic ground state in a flattened tetrahedron (Fig. 6). Therefore, the presence of this transition in the optical spectra is clear evidence of a JT distortion; in $T_{d}$ the corresponding transition energy, i.e., ${ }^{2} \mathrm{~T}_{2}$ splitting, would be zero besides splitting contributions caused by the spin-orbit interaction. The splitting of ${ }^{2} \mathrm{~B}_{2} \rightarrow{ }^{2} \mathrm{~A}_{1}+$ ${ }^{2} \mathrm{~B}_{1}$ is not observed spectroscopically since the transitions appear as a single band in the absorption spectrum due to band overlap and electric-dipole selection rules (Fig. 6). In fact, selection rules establish that within $D_{2 d}$ there are only two allowed electric-dipole transitions from the ${ }^{2} \mathrm{~B}_{2}$ ground state: ${ }^{2} \mathrm{~B}_{2} \rightarrow{ }^{2} \mathrm{E}$ ( $x, y$ polarized) and ${ }^{2} \mathrm{~B}_{2} \rightarrow{ }^{2} \mathrm{~A}_{1}$ ( $z$ polarized) [23], in agreement with experimental observations.

Figure 7 shows the peak energy variations of $d$ - $d$ transitions as a function of pressure in both Pnma and $\mathrm{HP}$ of $\mathrm{Cs}_{2} \mathrm{CuCl}_{4}$. Their transition energies and corresponding pressure rates are given in Fig. 7(b). Interestingly, the first JT-related band associated to ${ }^{2} \mathrm{~B}_{2} \rightarrow{ }^{2} \mathrm{E}$ transition shows a large redshift with pressure at a rate of $-73 \mathrm{meV} \mathrm{GPa}^{-1}$, while the second 
(a)

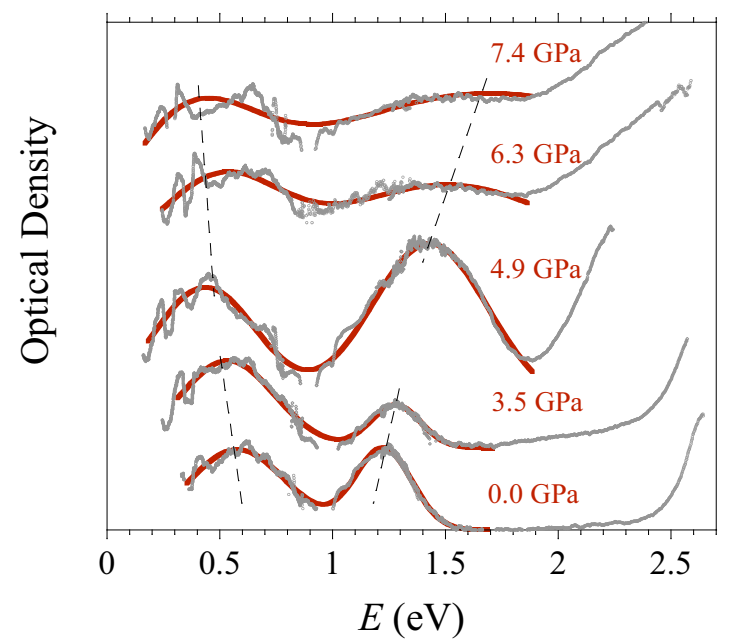

(b)

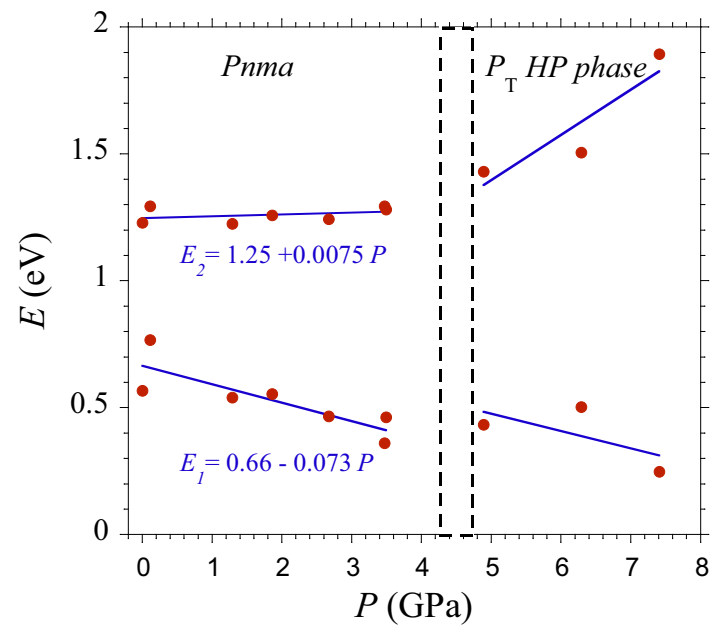

FIG. 7. (a) Variation of absorption spectra of $\mathrm{Cs}_{2} \mathrm{CuCl}_{4}$ with pressure in the Pnma and high-pressure phase $(P>4.9 \mathrm{GPa})$. (b) Variation of the peak energy of the main absorption bands with pressure. Pressure coefficients derived by fitting in the low-pressure Pnma phase are given in the least-squares linear fit equations.

one, associated to ${ }^{2} \mathrm{~B}_{2} \rightarrow{ }^{2} \mathrm{~A}_{1}$, shifts slightly toward higher energies $\left(+7.5 \mathrm{meV} \mathrm{GPa}^{-1}\right)$. It must be noted that the variation of $E(P)$ for both bands undergoes a change of slope at $4.9 \mathrm{GPa}$, thus supporting occurrence of a structural transformation in $\mathrm{Cs}_{2} \mathrm{CuCl}_{4}$ at this pressure, as it was unveiled by Raman spectroscopy. Given the sensitivity of $d$ - $d$ transitions to structural changes involving $\mathrm{Cu}^{2+}$ coordination change or variations of the bond distance and bond angle within $\mathrm{CuCl}_{4}^{2-}$, optical absorption becomes an adequate probe to explore phase-transition phenomena. It must also be noted that the absorption spectra was obtained in a powdered sample filling the whole pressure cavity to get suitable transmission spectra. However, this procedure yields nonhydrostatic effects that reduce the phase-transition pressure from $5.4 \mathrm{GPa}$ - single crystal in hydrostatic medium-to $4.9 \mathrm{GPa}$ - powder itself as pressure transmitting medium. Nevertheless, at a given pressure, the Raman spectra under these pressure conditions are identical for powder and single crystal.

The CT band gap is also very sensitive to pressure. $\mathrm{Cs}_{2} \mathrm{CuCl}_{4}$ is a CT semiconductor with a direct gap of $2.52 \mathrm{eV}$ which redshifts with pressure at a rate of $-20 \mathrm{meV} \mathrm{GPa}^{-1}$. It means that significant color changes are expected at pressure well above $5 \mathrm{GPa}$ as shown in Fig. 8. In fact, its strong piezochromism is a consequence of the pressure-induced band-gap redshift and mainly band broadening, which makes it to change the color with pressure from yellow-orange to black, particularly at the HP phase where band broadening drastically reduces the optical transmittance window (Fig. 8). To obtain the direct band gap, $E_{g}$, we have plotted $(h v \times \alpha)^{2}$ against $h v$, with $\alpha$ being the absorption coefficient. From the tail of the absorption threshold and once the background was subtracted, $E_{g}$ was obtained by the interception of the plot with $\alpha=0$. $E_{g}$ experiences an abrupt drop of about $0.3 \mathrm{eV}$ at the phase transition at $4.9 \mathrm{GPa}$, as we can see in Fig. 8(a). Above this pressure, the absorption spectra show a band structure with at least two strongly broadened bands at 0.43 and $1.43 \mathrm{eV}$, which are responsible for the crystal darkening (black color). Their pressure dependence is shown in Fig. 7. The Pnma phase is recovered in down-stroke below about $3 \mathrm{GPa}$, thus having a hysteresis of $2 \mathrm{GPa}$ at room temperature. As previously indicated, the difference between

(a)

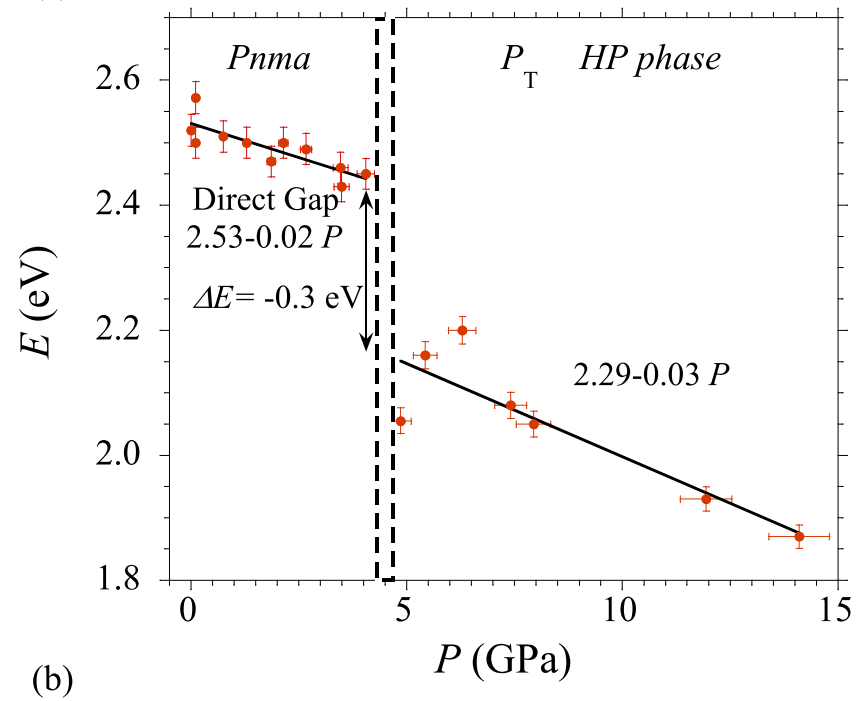

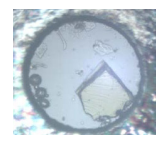

$0.1 \mathrm{GPa}$

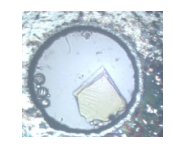

2.1 GPa

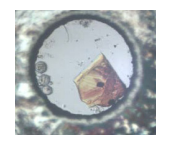

$5.4 \mathrm{GPa}$

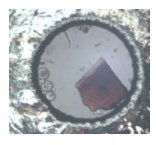

$7.9 \mathrm{GPa}$

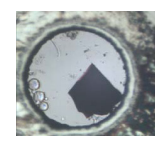

$18.2 \mathrm{GPa}$
FIG. 8. (a) Variation of the charge-transfer band gap with pressure in both Pnma phase and high-pressure phase. The direct gap redshifts with pressure. (b) Images of a single crystal of $\mathrm{Cs}_{2} \mathrm{CuCl}_{4}$ in a DAC at different pressures. Note how the crystal color varies from yellow to dark red and eventually to black upon increasing pressure. The piezochromism is associated with the redshifted CT band gap with pressure. Single crystal dimensions (ambient pressure): $80 \times$ $80 \times 22 \mu \mathrm{m}^{3}$. 
the transition pressure measured in single crystal (5.4 GPa) and powder (4.9 GPa) $\mathrm{Cs}_{2} \mathrm{CuCl}_{4}$ must be associated to a lack of hydrostaticity in powdered samples. However, the phase transition can be established at $4.9 \mathrm{GPa}$ in upstroke that corresponds to initial observation of traces of the HP phase within the pressure range of phase coexistence (Fig. 3).

\section{Angular overlap model for $\mathrm{CuCl}_{4}^{2-}$}

The angular overlap model (AOM) [24-27] is a powerful tool within the ligand-field theory that provides a semiquantitative explanation to the unusual pressure shifts of the two $d$ - $d$ bands (Fig. 7). The initial flattened-tetrahedron symmetry $\left(D_{2 d}\right)$ of $\mathrm{CuCl}_{4}^{2-}$, which splits the parent tetrahedral $t_{2}$ and $e$ orbitals into $b_{2}+e$ and $a_{1}+b_{1}$, respectively, will change when compressing $\mathrm{Cs}_{2} \mathrm{CuCl}_{4}$. The relative variations of the $T_{d}$ crystal-field strength and the JT-related $\mathrm{Cl}-\mathrm{Cu}-\mathrm{Cl}$ dihedral angle will evolve with pressure affecting the $d$-orbital splitting. According to crystal-field theory and experimental observations $[1,3]$, the crystal-field strength increases by decreasing the $\mathrm{Cu}-\mathrm{Cl}$ bond distance, $R$, whereas the dihedral angle tends to decrease with pressure, approaching the $T_{d}$ angle $\left(109.47^{\circ}\right)$ under high compression [26].
XRD results show that $R$ and $\gamma_{\mathrm{Cl}-\mathrm{Cu}-\mathrm{Cl}}$ change from $R=$ $2.230 \AA$ and $\gamma_{\mathrm{Cl}-\mathrm{Cu}-\mathrm{Cl}}=127.4^{\circ}$ at ambient pressure [25] to $R=2.199 \AA$ and $\gamma_{\mathrm{Cl}-\mathrm{Cu}-\mathrm{Cl}}=122.3^{\circ}$ at $3.9 \mathrm{GPa}$ [7] in agreement with expectations for a JT system [2]. Within AOM, $d$ - $d$ transition energies of $\mathrm{CuCl}_{4}^{2-}$ can be determined as a function of the structural parameter $\beta=1 / 2\left(\gamma_{\mathrm{Cl}-\mathrm{Cu}-\mathrm{Cl}}-\right.$ $109.47^{\circ}$ ), which represents the deviation of the $\mathrm{Cl}-\mathrm{Cu}-\mathrm{Cl}$ dihedral angle from its $T_{d}$ value. We use $\beta$ instead of the usual dihedral angle $\gamma_{\mathrm{Cl}-\mathrm{Cu}-\mathrm{Cl}}$ since $\beta=0$ in $T_{d}$ symmetry and $35.3^{\circ}$ in a square-planar $D_{4 h}$ symmetry. For $\mathrm{CuCl}_{4}^{2-}$ in $\mathrm{Cs}_{2} \mathrm{CuCl}_{4}, \beta=8.5 \pm 0.5^{\circ}$ at ambient conditions and $6.4 \pm$ $0.5^{\circ}$ at $3.9 \mathrm{GPa}$. AOM simulations provide an adequate tool for explaining variations of the $\mathrm{CuCl}_{4}^{2-}$ transition energies along compound series [24-27] but also as a function of pressure in $\mathrm{Cs}_{2} \mathrm{CuCl}_{4}$. In particular, $\mathrm{AOM}$ allows us to explain why the first band ${ }^{2} \mathrm{~B}_{2} \rightarrow{ }^{2} \mathrm{E}$ largely shifts to lower energy whereas the second one ${ }^{2} \mathrm{~B}_{2} \rightarrow{ }^{2} \mathrm{~A}_{1}$, more sensitive to the crystal-field strength, shifts slightly to higher energies with pressure.

Within $\mathrm{AOM}$, the electronic energies of a $\mathrm{Cu} X_{4}^{2-}$ system are given as a function of the AOM parameters $e_{\sigma}, e_{\pi}, e_{s d}$, and $e_{p d}$ and the $X-\mathrm{Cu}-X$ bond angle $\gamma$ as [27]

$$
\begin{aligned}
\Delta E\left({ }^{2} \mathrm{~B}_{2} \rightarrow{ }^{2} \mathrm{E}\right)= & 3\left[\sin ^{4}(\gamma / 2)-1 / 2 \sin ^{2}(\gamma)\right] e_{\sigma}+\left[\sin ^{2}(\gamma)-2 \cos ^{2}(\gamma)-2 \cos ^{2}(\gamma / 2)\right] e_{\pi}, \\
\Delta E\left({ }^{2} \mathrm{~B}_{2} \rightarrow{ }^{2} \mathrm{~B}_{1}\right)= & 3 \sin ^{4}(\gamma / 2) e_{\sigma}+\left[\sin ^{2}(\gamma)-4 \sin ^{2}(\gamma / 2)\right] e_{\pi}-13.3 \sin ^{4}(\gamma / 2) \cos ^{2}(\gamma / 2) e_{p d}, \\
\Delta E\left({ }^{2} \mathrm{~B}_{2} \rightarrow{ }^{2} \mathrm{~A}_{1}\right)= & 3 \sin ^{4}(\gamma / 2) e_{\sigma}-4\left[\cos ^{2}(\gamma / 2)-1 / 2 \sin ^{2}(\gamma / 2)\right]^{2} e_{\sigma}-2 \sin ^{2}(\gamma) e_{\pi} \\
& +16\left[\cos ^{2}(\gamma / 2)-1 / 2 \sin ^{2}(\gamma / 2)\right]^{2} e_{s d}-13.3 \sin ^{4}(\gamma / 2) \cos ^{2}(\gamma / 2) e_{p d} .
\end{aligned}
$$

These expressions are suitable to account for the transition energies in different $\mathrm{Cu} X_{4}^{2-}$ systems having different dihedral angles [25]. This has been especially useful for explaining the variation of transition energies obtained from spectroscopic data as a function of the dihedral angle in $\mathrm{Cu}^{2+}$ chlorides providing dihedral angles for $\mathrm{CuCl}_{4}^{2-}$ ranging from $127^{\circ}$ to $180^{\circ}$ or, equivalently, from $\beta=8.5^{\circ}$ to $\beta=35.3^{\circ}$ [24-27]. The spectroscopic series of $\mathrm{CuCl}_{4}^{2-}$ can be explained using the following AOM parameters: $e_{\sigma}=0.635 \mathrm{eV}, e_{\pi}=0.113 \mathrm{eV}$, $e_{s d}=0.114 \mathrm{eV}$, and $e_{p d}=-0.0025 \mathrm{eV}$ [25-27]. Figure 9 shows the energy of the $d$ - $d$ transitions of $\mathrm{CuCl}_{4}^{2-}$ as a function of $\beta$, where additionally we have included the spin-orbit interaction $\left(\lambda=-829 \mathrm{~cm}^{-1}\right)$ [25].

To simulate the pressure-induced energy shifts in $\mathrm{Cs}_{2} \mathrm{CuCl}_{4}$, we have calculated the transition energies for $\mathrm{CuCl}_{4}^{2-}$ using the AOM parameters at ambient pressure with $\beta=8.5^{\circ}$, and at $3.9 \mathrm{GPa}$ with $\beta=6.4^{\circ}$ and scaling the AOM parameters following a potential law for the bond distance as $\left(R_{0} / R\right)^{5}$ using the equation of state of $\mathrm{Cs}_{2} \mathrm{CuCl}_{4}$ in the Pnma phase [7]. The so-obtained AOM parameters at $3.9 \mathrm{GPa}$ are $e_{\sigma}=0.78 \mathrm{eV}, e_{\pi}=0.139 \mathrm{eV}, e_{s d}=0.172 \mathrm{eV}$, and $e_{p d}=$ $-0.0031 \mathrm{eV}$. The results of these simulations allow us to explain the band shifts (Fig. 9). $R$ reduction due to pressure increases the energy separation of the parent tetrahedral orbitals, $e$ and $t_{2}$, by $10 \mathrm{Dq}$, while reduction of $\beta$ decreases the $t_{2}$ splitting in $D_{2 d}$. Both effects induce band shifts similar to those observed experimentally (Fig. 9).
These structural correlations, which are based on the energy shifts of the crystal-field bands in $\mathrm{Cs}_{2} \mathrm{CuCl}_{4}$, indicate that the main structural effect of the JT-flattened $\mathrm{CuCl}_{4}^{2-}$ is reducing the $\mathrm{Cl}-\mathrm{Cu}-\mathrm{Cl}$ bond angle reduces from $8.5^{\circ}$ to $6.4^{\circ}$ at 3.9 GPa consistently with structural data [7], and therefore supports the adequacy of the $d$ - $d$ spectra to explore structural changes induced by pressure in transition-metal chlorides involving $\mathrm{JT}$ ions like $\mathrm{Cu}^{2+}$.

\section{CONCLUSIONS}

We have demonstrated that the vibrational and electronic structures of $\mathrm{Cs}_{2} \mathrm{CuCl}_{4}$ can be explained to a great extent on the basis of $\mathrm{CuCl}_{4}^{2-}$ with a $D_{2 d}$ coordination symmetry (flattened tetrahedron), their pressure dependence being consistent with structural changes undergone by $\mathrm{Cs}_{2} \mathrm{CuCl}_{4}$. The two intense $D_{2 d}$-allowed $d$ - $d$ bands behave differently with pressure, the pressure coefficient of which being consistent with a reduction of both the $\mathrm{Cu}-\mathrm{Cl}$ bond distance and the $\mathrm{Cl}-\mathrm{Cu}-\mathrm{Cl}$ bond angle, i.e., reduction of the JT distortion with pressure, in agreement with previous XRD data. The AOM fairly accounts for the their pressure shifts, according to proposed structural scenario.

We showed that the piezochromism of $\mathrm{Cs}_{2} \mathrm{CuCl}_{4}$ in the Pnma phase is governed by the pressure-induced redshift of the CT band gap that reduces the spectral optical window of transmittance yielding a progressive color change from yellow to orange in the $0-5 \mathrm{GPa}$ range. The strong piezochromic 


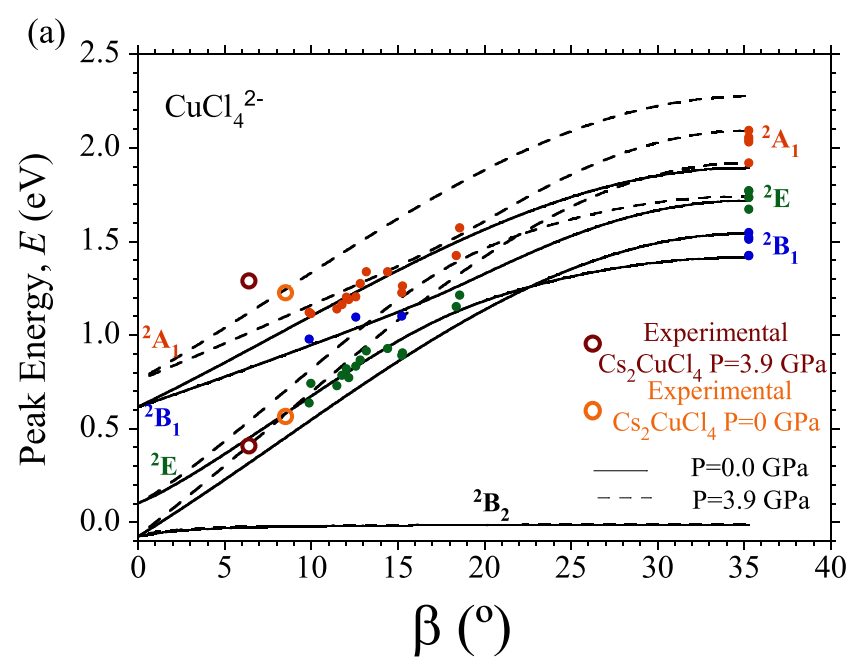

(b)
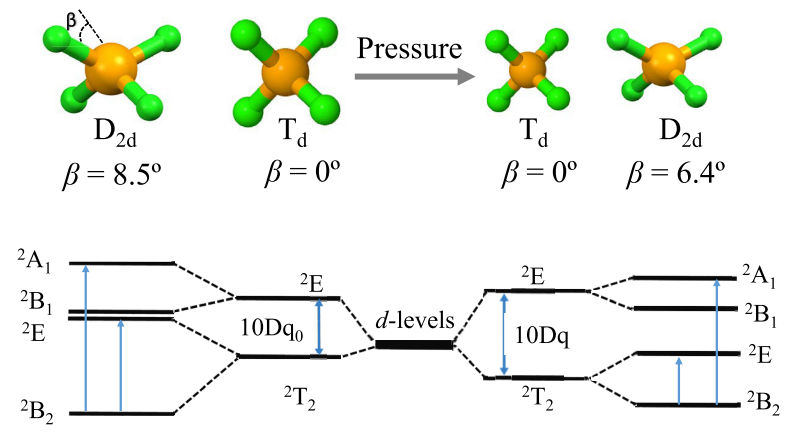

FIG. 9. (a) Calculated crystal-field energies for $\mathrm{CuCl}_{4}^{2-}$ as a function of the distortion angle $(\beta)$ using AOM. $\beta=0^{\circ}$ corresponds to $T_{d}$ (regular tetrahedron) and $\beta=32.5^{\circ}$ to $D_{4 h} \mathrm{CuCl}_{4}^{2-}$ (square-planar coordination). Solid and dashed lines correspond to calculations at ambient pressure and $3.9 \mathrm{GPa}$, respectively. The spin-orbit coupling have been included in the calculations with $\lambda=-829 \mathrm{~cm}^{-1}$ (see text for details). Filled colored symbols correspond to experimental data from compound series providing different $\mathrm{Cl}-\mathrm{Cu}-\mathrm{Cl}$ bond angles for $\mathrm{CuCl}_{4}^{2-}[24,25]$. Empty circles correspond to present experimental data for $\mathrm{Cs}_{2} \mathrm{CuCl}_{4}$ at ambient pressure and $3.9 \mathrm{GPa}$. Note that the pressure-induced energy variations are in agreement with structural data obtained by XRD [7]. (b) Schematic diagram of the $\mathrm{Cu}^{2+} d$-orbital splitting in a tetrahedron ( $\left.T_{d}\right)$ and a flattened tetrahedron $\left(D_{2 d}\right)$ for four different configurations corresponding to ambient pressure (left side) and high-pressure conditions (right side). In $T_{d}$, only $R$ is changed whereas both $R$ and $\beta$ are modified in $D_{2 d}$.

effect accompanying the structural phase transition to a highly amorphized phase at $5.4 \mathrm{GPa}$ is associated with an additional CT redshift but mainly to band broadening that reduces the optical transmission window of the crystal yielding crystal darkening. Crystal darkness increases with pressure becoming black at $18.2 \mathrm{GPa}$. The new HP phase probably involves a change of $\mathrm{Cu}^{2+}$ coordination from $\mathrm{CuCl}_{4}^{2-}$ flattened tetrahedra to a more compact structure consisting of ligand-sharing $\mathrm{CuCl}_{6}^{4-}$ octahedra as suggested by the Raman and $d$ - $d$ absorption spectra. These links increase $d$-orbital overlap between neighboring $\mathrm{Cu}^{2+}$, thus favoring band broadening. However, the HP phase structure lacks from being yet resolved.

We have also demonstrated that the large thermal redshift of the totally symmetric breathing $v_{1}\left(A_{1}\right)$ mode of $\mathrm{Cs}_{2} \mathrm{CuCl}_{4}$ in the $10-600 \mathrm{~K}$ range is governed by thermal expansion and anharmonic phonon-phonon coupling effects, their relative contribution being of $68 \%$ and $32 \%$, respectively. The thermal-expansion intrinsic contribution to the thermal shift has been described through the measured pressure coefficient, whereas the anharmonic phonon-coupling extrinsic contribution has been modeled by two- and three-phonon coupling interactions, both having similar contributions.

A salient conclusion is the suitability of performing combined temperature and pressure dependence measurements of the vibrational and electronic peaks in order to unveil the different microscopic mechanisms responsible for the pressure and temperature induced shifts.

\section{ACKNOWLEDGMENTS}

Financial support from the Spanish Ministerio de Economía, Industria y Competitividad (MAT2015-69508-P) and MALTA-CONSOLIDER (MAT2015-71010REDC). E.J. thanks the Spanish Ministerio de Ciencia, Innovación y Universidades for a FPI research Grant (BES-2016-077449).
[1] H. G. Drickamer and K. L. Bray, Acc. Chem. Res. 23, 55 (1990).

[2] J. Ferguson, J. Chem. Phys. 40, 3406 (1964).

[3] L. Nataf, F. Aguado, I. Hernández, R. Valiente, J. González, M. N. Sanz-Ortiz, H. Wilhelm, A. P. Jephcoat, F. Baudelet, and F. Rodríguez, Phys. Rev. B 95, 014110 (2017).

[4] D. Machon, A. Grzechnik, and K. Friese, J. Phys.: Condens. Matter. 21405405 (2009).

[5] O. Lamba and S. Sinha, Sol. St. Commun. 57, 365 (1986).

[6] J. A. McGinnety, J. Am. Chem. Soc. 94, 8406 (1972).

[7] Y. Xu, S. Carlson, K. Söderberg, and R. Norrestam, J. Solid State Chem. 153, 212 (2000).
[8] H. Yi, F. Xia, Q. Zhou, and D. Zeng, J. Phys. Chem. A 115, 4416 (2011).

[9] R. Valiente and F. Rodríguez, J. Phys.: Condens. Matter 10, 9525 (1998).

[10] S. Klotz, J.-C. Chervin, P. Munsch, and G. L. Marchand, J. Phys. D: Appl. Phys. 42, 075413 (2009).

[11] B. A. Moral and F. Rodríguez, Rev. Sci. Instrum. 66, 5178 (1995).

[12] K. Syassen, High Pressure Res. 28, 75 (2008).

[13] J. Barreda-Argüeso and F. Rodríguez, Patent No. PCT/ES2014/000049 (2015). 
[14] H. Mashiyama and N. Koshiji, Acta Crystallogr. B: Struct. Sci. 45, 467 (1989).

[15] I. R. Beattie, T. R. Gilson, and G. A. Ozin, J. Chem. Soc. A: Inorganic, Physical, Theoretical 534 (1969).

[16] K. Nakamoto, Infrared and Raman Spectra of Inorganic and Coordination Compounds ( Wiley, Michigan, 1963).

[17] F. Decremps, J. Pellicer-Porres, A. M. Saitta, J. C. Chervin, and A. Polian, Phys. Rev. B 65, 092101 (2002).

[18] M. M. de Lucas, F. Rodriguez, and M. Moreno, J. Phys.: Condens. Matter 7, 7535 (1995).

[19] M. Balkanski, R. F. Wallis, and E. Haro, Phys. Rev. B 28, 1928 (1983).

[20] J. Menéndez and M. Cardona, Phys. Rev. B 29, 2051 (1984).

[21] Z. Tylczyński, P. Piskunowicz, A. N. Nasyrov, A. D. Karaev, K. T. Shodiev, and G. Gulamov, Phys. Status Solidi A 133, 33 (1992).
[22] J. S. Griffith, The theory of Transition-Metal Ions (Cambridge University Press, Cambridge, 1980).

[23] R. Valiente and F. Rodríguez, J. Phys.: Condens. Matter 11, 2595 (1999).

[24] M. A. Hitchman, Comments Inorg. Chem. 15, 197 (1994).

[25] M. Atanasov, D. Ganyushin, K. Sivalingam, and F. Neese, A modern first-principles view on ligand field theory through the eyes of correlated multireference wavefunctions, in Molecular Electronic Structures of Transition Metal Complexes II, edited by D. M. P. Mingos, P. Day, and J. P. Dahl (Springer Berlin, 2012), pp. 149-220.

[26] M. Atanasov, B. Delley, and D. Reinen, Anorg. Allg. Chem. 636, 1740 (2010).

[27] R. G. McDonald, M. J. Riley, and M. A. Hitchman, Inorg. Chem. 27, 894 (1988). 